\title{
CCL25 mediates migration, invasion and matrix metalloproteinase expression by breast cancer cells in a CCR9-dependent fashion
}

\author{
CRYSTAL JOHNSON-HOLIDAY ${ }^{1}$, RAJESH SINGH ${ }^{1}$, ERICA JOHNSON ${ }^{1}$, SHAILESH SINGH $^{1}$, \\ CECIL R. STOCKARD ${ }^{2}$, WILLIAM E. GRIZZLE ${ }^{2}$ and JAMES W. LILLARD Jr${ }^{1}$ \\ ${ }^{1}$ Department of Microbiology, Biochemistry and Immunology, Morehouse School of Medicine, Atlanta, GA; \\ ${ }^{2}$ Department of Pathology, University of Alabama at Birmingham, School of Medicine, Birmingham, AL, USA
}

Received September 6, 2010; Accepted October 4, 2010

DOI: 10.3892/ijo.2011.953

\begin{abstract}
Breast cancer $(\mathrm{BrCa})$ is one of the most frequently diagnosed cancers and the second leading cause of cancerrelated deaths in North American women. Most deaths are caused by metastasis, and $\mathrm{BrCa}$ is characterized by a distinct metastatic pattern involving lymph nodes, bone marrow, lung, liver and brain. Migration of metastatic cells share many similarities with leukocyte trafficking, which are regulated by chemokines and their receptors. The current study evaluates the expression and functional role of CCR9, and its only known ligand, CCL25, in BrCa cell migration and invasion. Quantitative immunohistochemical analysis showed that both moderately and poorly differentiated $\mathrm{BrCa}$ tissue expressed significantly more $(\mathrm{P}<0.0001)$ CCR 9 compared to non-neoplastic breast tissue. Interestingly, poorly differentiated $\mathrm{BrCa}$ tissue expressed significantly more $(\mathrm{P}<0.0001) \mathrm{CCR} 9$ compared to moderately differentiated BrCa tissue. Similarly, CCR9 was highly expressed by the aggressive breast cancer cell line (MDA-MD-231) compared to the less aggressive MCF-7. Migration as well as invasion assays were used to evaluate the functional interaction between CCR9 and CCL25 in BrCa cell lines (MDA-MB-231 and MCF-7). Neutralizing CCR9-CCL25 interactions significantly impaired the migration and invasion of $\mathrm{BrCa}$ cells. Furthermore, CCL25 enhanced the expression of MMP-1, -9, -11 and -13 active proteins by $\mathrm{BrCa}$ cells in a CCR9dependent fashion. These studies show CCR9 is functionally and significantly expressed by $\mathrm{BrCa}$ (poorly $>$ moderately differentiated) tissue and cells as well as that CCL25 activation of this receptor promotes breast tumor cell migration, invasion and MMP expression, which are key components of BrCa metastasis.
\end{abstract}

Correspondence to: Dr James W. Lillard Jr, Morehouse School of Medicine, 720 Westview Drive SW, Atlanta, GA 30310-1495, USA E-mail: jlillard@msm.edu

Key words: chemokine, immunobiology, matrix metalloproteinase

\section{Introduction}

In the United States, $\mathrm{BrCa}$ is the most common non-skin cancer and the second leading cause of cancer deaths in women, behind lung cancer (1). Metastasis is responsible for the majority of BrCa-related deaths. The development of metastasis consists of a complex series of sequential steps (2). These processes include invasion through extracellular matrix, intravasation, survival in the circulation, extravasation into a distant site, and progressive growth at the site of metastasis (3). BrCa progression is characterized by a distinct metastatic pattern involving regional lymph nodes, bone marrow, lung, brain and liver (4).

Many factors have been implicated in the process of metastasis, but the precise mechanisms for the directional migration of malignant cells to different organs are incompletely known. This process shares many similarities with leukocyte trafficking, which is largely mediated by chemokines. In this regard, chemokines are a super family of small, cytokine-like proteins that induce, through their interaction with $\mathrm{G}$ protein-coupled receptors, cytoskeletal rearrangement, firm adhesion to endothelial cells, and directional migration. These secreted proteins act in a coordinated fashion with cell-surface proteins, including integrins, to direct the specific homing of various subsets of cells. It has been shown that CXCL12-CXCR4 interactions play a role in the metastasis of $\mathrm{BrCa}$ cells to regional lymph nodes and lung (5). It has also been demonstrated that neoplastic breast tissues express higher levels of CXCR4 than normal breast tissue.

CXCR4/CXCL12 interactions alone do not fully explain the pattern of $\mathrm{BrCa}$ metastasis or the potential of $\mathrm{BrCa}$ cells to migrate and invade other tissues. In this study, we have tested the hypothesis that breast carcinomas partially use CCR9-dependent mechanisms for migration, invasion and matrix metalloproteinase (MMP) expression. To test this hypothesis, we evaluated the levels of CCR9 mRNA and surface protein expression by $\mathrm{BrCa}$ cell lines and tissue. The functional relevance of this expression was demonstrated using migration, extracellular matrix (ECM) invasion and MMP expression assays. These studies suggest the expression of CCR9 and its interaction with CCL25 aid in BrCa cell migration, invasion and MMP expression to support the 
differential metastatic potential of moderately differentiated versus poorly differentiated $\mathrm{BrCa}$.

\section{Material and methods}

Breast cancer tissue microarray. BrCa tissue microarrays were obtained from the Southern Division of the Cooperative Human Tissue Network (CHTN), the University of Alabama at Birmingham and Accumax Ltd. To construct these tissue microarrays, at least two cores (1 $\mathrm{mm}$ in diameter) per patient were arrayed on a receiver blank paraffin block. A qualified pathologist characterized and validated the histopathology, class and grade of each tumor or benign tissue core. The breast tissue microarrays used in this study were composed of tumors from 36 patients. These breast tumors represented distinct histopathological subtypes (18 moderately differentiated and 18 poorly differentiated as well as 9 non-neoplasticmatched benign tissues. The tissue microarray was cut in $4-\mu \mathrm{m}$ sections and placed on super frost-charged glass microscope slides.

Quantitation of immunohistochemical staining. To numerically analyze the immunohistochemical staining, virtual slides were created from stained samples after scanning each specimen using an Aperio ScanScope scanning system (Aperio Technologies). A color markup image for each slide was obtained based on membrane staining. The ScanScope generated true color digital images of each stained sample, which were viewed using Aperio ImageScope version 6.25 software. The ImageScope algorithm for determining the intensity of membrane-specific staining was used to calculate the staining intensity and percent target label for each sample by digitally analyzing the color intensity. The output of stain intensities ranging from 0 to 3 correlated with conventional manual scoring methods (where 0 , negative and 3 , strong staining).

Cell lines and cell culture. BrCa cell lines (MDA-MB-231 and MCF-7) were obtained from ATCC. MDA-MB-231 and $\mathrm{MCF}-7$ cell lines were maintained in $5 \% \mathrm{CO}_{2}$ at $37^{\circ} \mathrm{C}$ in RPMI-1640 media supplemented with $10 \%$ fetal bovine serum, L-glutamine, sodium pyruvate, non-essential amino acids and antibiotics (Sigma-Aldrich). Before migration and invasion studies, $\mathrm{BrCa}$ cells were cultured for $24 \mathrm{~h}$ in RPMI1640 with $2 \%$ FBS.

RNA isolation and RT-qPCR analysis. Total RNA was isolated from untreated and CCL25-treated BrCa cells using Tri-Reagent (Molecular Research Center) according to the manufacturer's protocol. Potential genomic DNA contamination was removed from these samples by treatment with RNase-free DNase (Invitrogen). RNA was precipitated and resuspended in RNA Secure (Ambion). Complementary DNA was generated by reverse transcribing $1.5 \mu \mathrm{g}$ of total RNA using iScript cDNA Synthesis kit (BioRad) according to manufacturer's protocols, and amplified with specific cDNA primers using iQ SYBR-Green Supermix (BioRad). The number of mRNAs relative to $18 \mathrm{~S}$ rRNA copies of these targets was evaluated by RT-PCR analysis using the Bio-Rad Icycler and software.
Primer design. Human mRNA sequences for CCR9, MMP-1, MMP-2, MMP-3, MMP-9, MMP-10, MMP-11, MMP-13 and $18 \mathrm{~S}$ rRNA were obtained from the National Institutes of Health National Center for Biotechnology Information GenBank database accession nos. XM003251, NM002421, NM004530, NM002422, NM004994, NM005940, NM002426, NM002427 and X00686.1, respectively. Primers were designed using the Primer 3 software program from the Whitehead Institute at Massachusetts Institute of Technology. These sequences were then used to design primers for RTPCR analysis, which generated amplicons of 162, 83, 95, 155, 79, 94, 107, 176 and 149 bp in size for CCR9, MMP-1, MMP-2, MMP-3, MMP-9, MMP-10, MMP-11, MMP-13 and $18 \mathrm{~S}$ rRNA, respectively.

ImageStream analysis of CCR9 expression. Fluorescein isothiocynate (FITC)-conjugated mouse anti-human CCR9 antibody was purchased from R\&D Systems. FITC-conjugated mouse IgG2a monoclonal antibody isotype control was obtained from PharMingen. BrCa cell lines were washed three times in PBS supplemented with $0.5 \%$ bovine serum albumin (BSA), and treated with $1.0 \mu \mathrm{g}$ of human IgG per $10^{5}$ cells for $15 \mathrm{~min}$ at room temperature. Fc-blocked $\mathrm{BrCa}$ cells were stained with $1.0 \mu \mathrm{g}$ of FITC-conjugated mouse antihuman CCR9 or FITC-conjugated mouse IgG2a isotype control antibody per $10^{5}$ cells at $4^{\circ} \mathrm{C}$ for $40 \mathrm{~min}$. Afterwards, cells were washed with $1.0 \mathrm{ml}$ of fluorescence-activated cell sorting buffer (1\% BSA in PBS) to remove unbound antibodies. Next, labeled cells were fixed with $500 \mu 1$ of $2 \%$ paraformaldehyde solution, and $10^{5}$ cells were analyzed by multispectral imaging flow cytometry using ImageStream system and IDEAS 3.0 software (Amnis).

Migration and invasion assays. CCL25 was obtained from PeproTech. Unlabeled mouse anti-human CCR9 antibodies were purchased from R\&D Systems. Migration and invasion studies were performed using BD Biocoat migration or Matrigel invasion chambers (Becton-Dickson Labware), respectively. RPMI-1640 was added to the interior of the bottom and top chamber of inserts and allowed to hydrate for $2 \mathrm{~h}$ at $37^{\circ} \mathrm{C}$ with $5 \% \mathrm{CO}_{2}$. CCL25 $(100 \mathrm{ng} / \mathrm{ml})$ was added to the bottom chamber. Next $10^{4}$ cells were added to the top chamber of inserts. To block chemokine receptor interaction, $1.0 \mu \mathrm{g} / \mathrm{ml}$ of mouse anti-human CCR9 (R\&D Systems) was added to the top chamber of Matrigel or control inserts and incubated overnight at $37^{\circ} \mathrm{C}$ and $5 \% \mathrm{CO}_{2}$. After incubation, cells at the bottom surface of the insert were fixed with $100 \%$ methanol for $2 \mathrm{~min}$, stained for 2 min with $1 \%$ toluidine blue (Sigma) supplemented with $1 \%$ borax (Sigma), and rinsed twice with deionized water. Cells were counted by microscopy at $\mathrm{x} 40$ magnification.

Active matrix metalloproteinase (MMP) protein detection. BrCa cells $\left(10^{5}\right)$ were seeded in 12-well plates, and conditioned media were collected from CCL25 (100 ng/ml)-treated or untreated cells. Active collagenases (MMP-1 and MMP-13), gelatinases (MMP-2 and MMP-9) and stromelysins (MMP-3, MMP-10 and MMP-11) levels were measured by Fluorokine (R\&D Systems) or Biotrak (GE Healthcare) assays, according to manufacturer's protocols. 

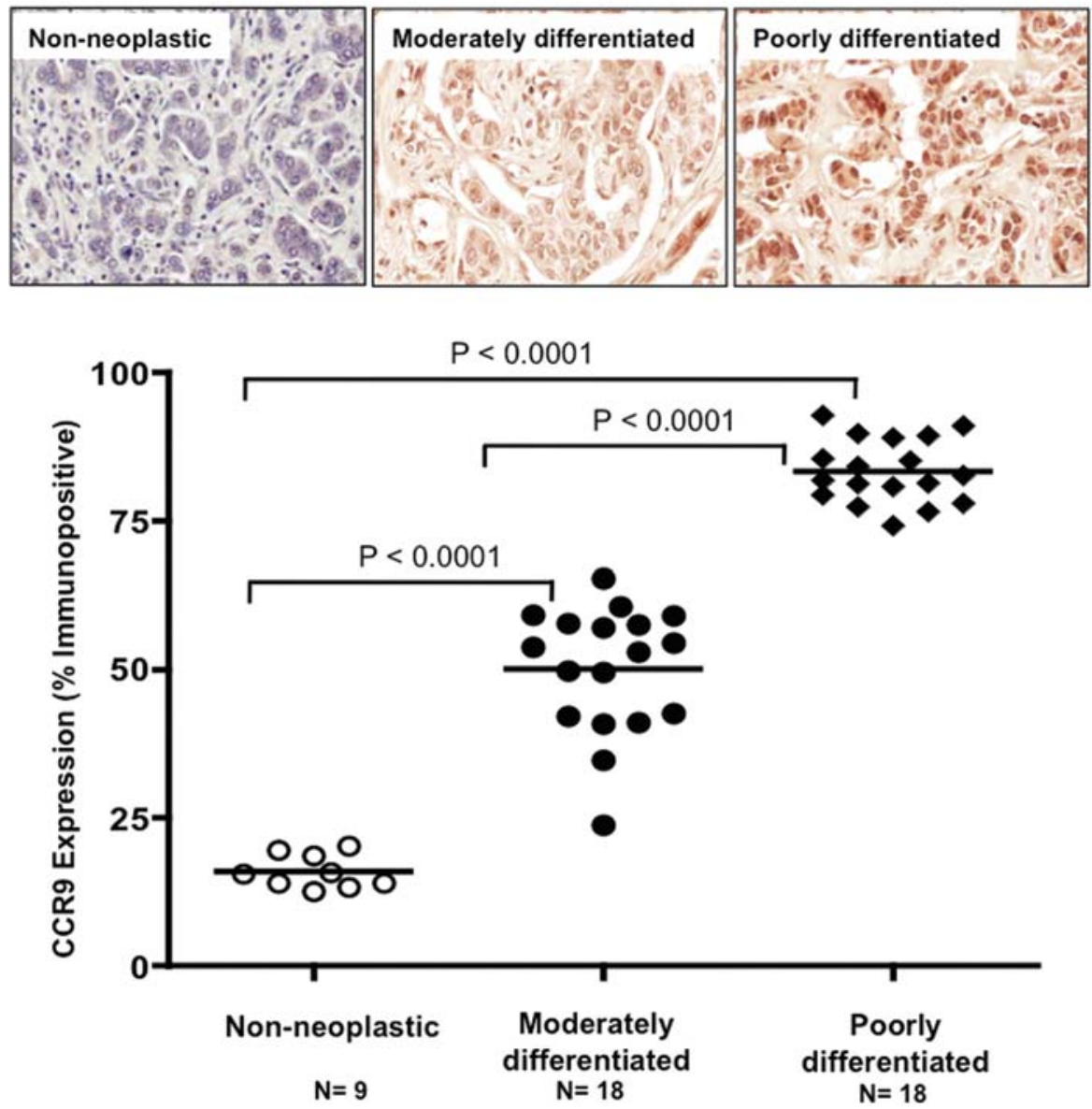

Figure 1. CCR9 expression by non-neoplastic mammary and breast cancer tissue. Breast tissues were stained with isotype control or anti-CCR9 antibodies Brown (DAB) color shows CCR9 staining. An Aperio ScanScope CS system with a x40 objective-captured digital images of each slide. Representative cases are indicated and immuno-intensities of CCR9 were quantified using image analysis Aperio ImageScope v.6.25 software. The percentages of CCR9 immunopositive malignant cells were determined for non-neoplastic matched benign $(0 ; n=9)$ as well as moderately $(\bullet ; n=18)$ and poorly $(\bullet ; n=18)$ differentiated carcinomas. Statistical significance was determined using unpaired t-test, comparing moderately to poorly differentiated breast carcinomas or non-neoplastic to BrCa tissues.

A

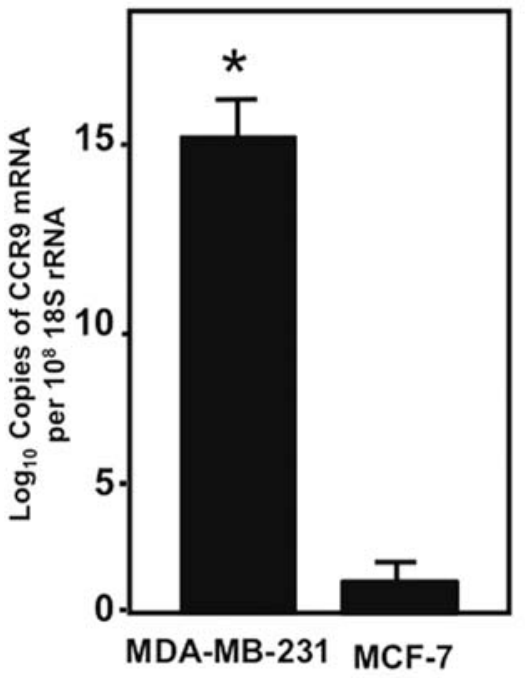

B
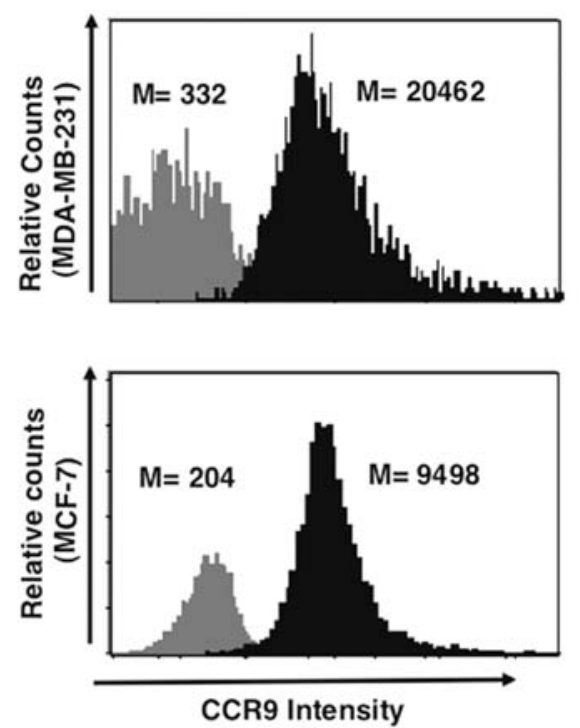

C
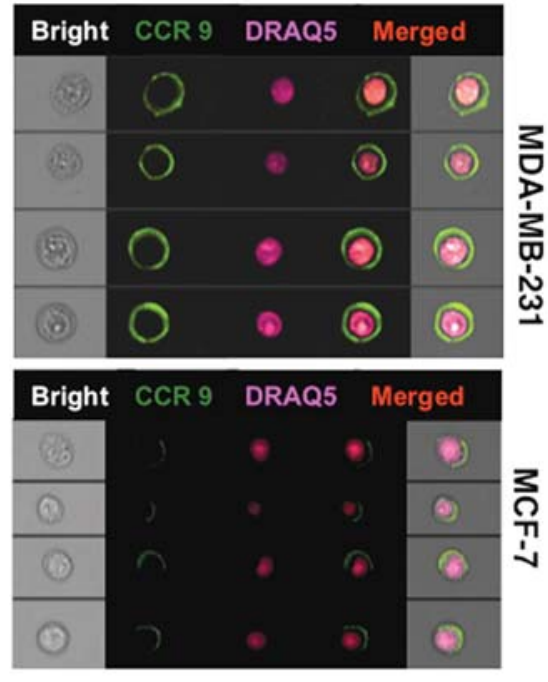

Figure 2. CCR9 expression by breast cancer cell lines. (A) Total RNA was isolated from MCF-7 and MDA-MB-231 cell lines, and quantitative RT-PCR analysis of CCR9 mRNA expression was performed in triplicate. The copies of transcripts are expressed relative to actual copies of $18 \mathrm{~S}$ rRNA \pm SE. ${ }^{*}$ Statistical significance $(\mathrm{p}<0.01)$ between normal tissue and BrCa cells. (B) MDA-MB-231 and MCF-7 cells were stained with FITC-conjugated isotype control antibodies (solid histogram) or anti-CCR9 monoclonal antibodies (open histogram) and quantified by flow cytometry. The mean fluorescent intensities (M) of CCR9-positive cells are shown. 

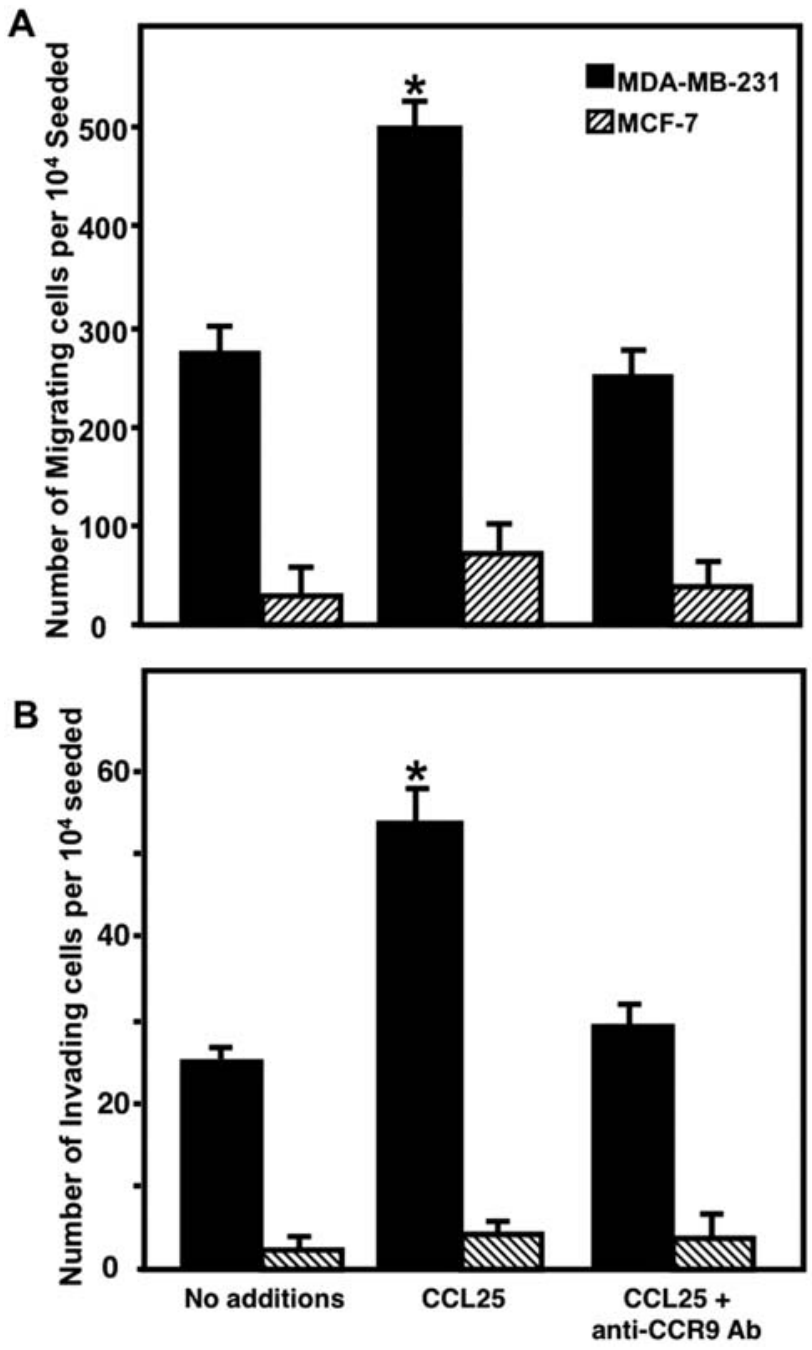

Figure 3. CCR9-mediated breast cancer cell lines migration and invasion. (A) MDA-MB-231 and MCF-7 cells were tested for their ability to migrate toward chemotactic gradients of CCL25. Cells were co-cultured with $1.0 \mu \mathrm{g} / \mathrm{ml}$ mouse anti-CCR9 antibody during migration assays using $100 \mathrm{ng} / \mathrm{ml}$ of CCL25. (B) MDA-MB-231 and MCF-7 cells were also tested for their ability to invade or translocate across Matrigel matrix in response to $100 \mathrm{ng} / \mathrm{ml}$ of CCL25. Cells were co-cultured with $1.0 \mu \mathrm{g} / \mathrm{ml}$ of control antibodies or monoclonal antibodies against CCR9 during invasion assays using $100 \mathrm{ng} / \mathrm{ml}$ of CCL25. The number of cell $( \pm$ SE) that migrated or invaded is shown with ${ }^{*}$ that indicate significant differences between no additions and chemokine-induced cells.

Statistical analysis. Data are expressed as the mean \pm SEM (standard error of mean) and compared using a two-tailed Student's t-test or an unpaired Mann-Whitney U-test. The results were analyzed using the GraphPad Prism software (GraphPad Software Inc., La Jolla, CA, USA) and were considered statistically significant at $\mathrm{P}<0.01$. When levels were below the detectable limit, values were recorded as onehalf of the lower detection limit (e.g., $50 \mathrm{pg} / \mathrm{ml}$ for active MMP-13) for statistical analysis. The Kolmogorov-Smirnov (K-S) two-sample test using IDEAS Software (Amnis) was used to compute the statistically significant differences between CCR9 histograms.

\section{Results}

CCR9 expression by BrCa tissue and cell lines. Representative samples of non-neoplastic mammary tissue as well as
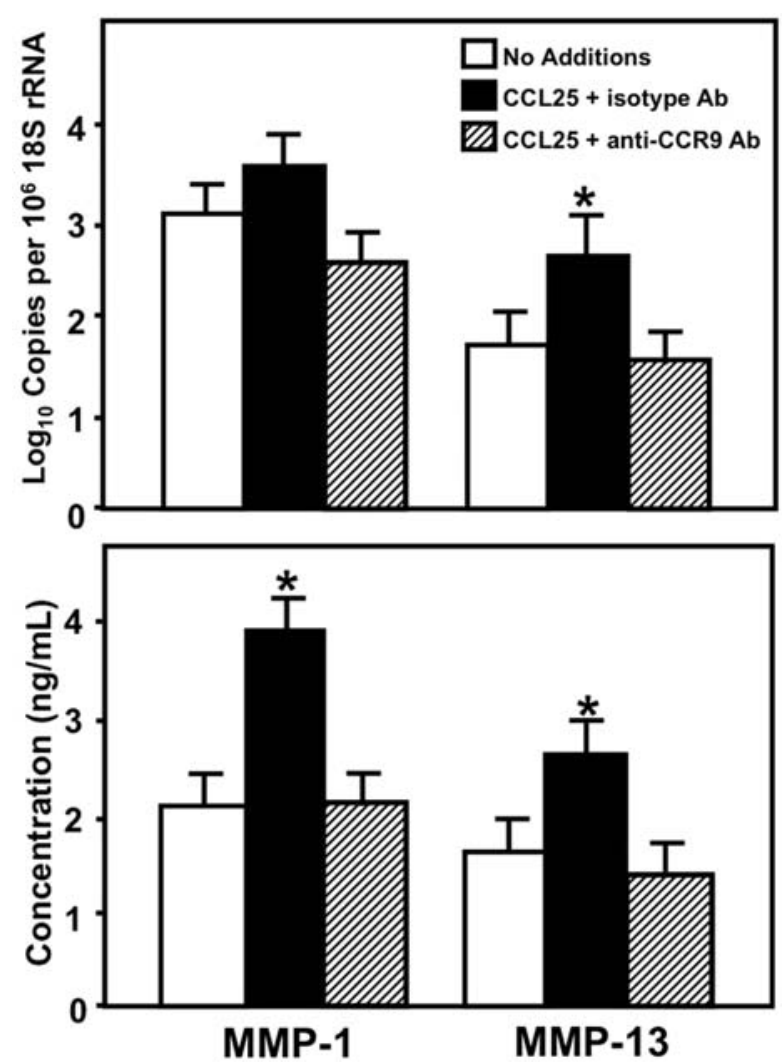

Figure 4. CCL25-induced collagenase expression by breast cancer cells. Cells were tested for their ability to express collagenases (MMP-1 and MMP-13) mRNA and active protein. MDA-MB-231 cells were cultured for $24 \mathrm{~h}$ alone, with $100 \mathrm{ng} / \mathrm{ml}$ of CCL25 or CCL25 $+1.0 \mu \mathrm{g} / \mathrm{ml}$ of mouse anti-CCR9 antibody. Total RNA was isolated and quantitative RT-PCR analysis was performed for mRNA expression of MMPs and transcript copies are presented relative to actual copies of $18 \mathrm{~S}$ rRNA. Active protein expression was quantified by Fluorokine and Biotrak assays in conditioned media. *Statistical differences $(\mathrm{P}<0.01)$ between untreated and CCL25-treated BrCa cells.

moderately and poorly differentiated BrCa specimens show differential CCR9 staining levels (Fig. 1). BrCa specimens expressed significantly higher levels of CCR9, than nonneoplastic, matched-benign tissue. The strongest CCR9 expression was found in the poorly differentiated cancer specimens. The approximate percentages of immunopositive malignant cells were determined for 14 cases of moderately and 14 poorly differentiated carcinomas. Bars, mean percentagepositivity show the poorly differentiated carcinomas express significantly higher CCR9. Statistical significance was determined by unpaired t-test for comparing moderately to poorly differentiated carcinoma. MDA-MB-231 and MCF-7 cell lines expressed CCR9 mRNA; however, significantly higher levels of CCR9 mRNA were observed in MDA-MB231 cells than compared to MCF-7 cells (Fig. 2). As with mRNA expression, significant surface protein expression of CCR9 by MDA-MB-231 cells was confirmed by multispectral imaging flow cytometry. The surface protein expression of CCR9 by MDA-MB-231 cells was significantly higher than MCF-7 cells.

CCL25-mediated migration and invasion. The functional significance of CCR9 expression was demonstrated by the ability of MDA-MB-231 cells to migrate toward chemotactic 

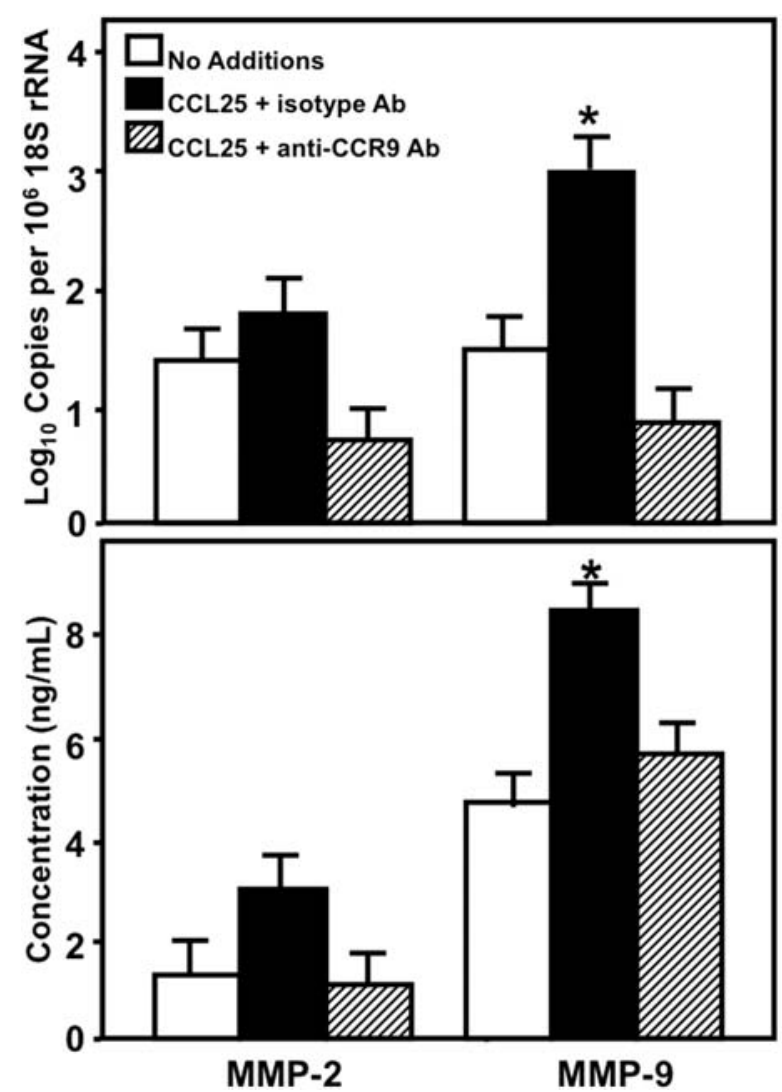

Figure 5. CCL25-induced gelatinase expression by breast cancer cells. Cells were tested for their ability to express gelatinases (MMP-2 and MMP-9) mRNA and active protein. MDA-MB-231 cells were cultured for $24 \mathrm{~h}$ alone, with $100 \mathrm{ng} / \mathrm{ml}$ of CCL25 or CCL25 $+1.0 \mu \mathrm{g} / \mathrm{ml}$ of mouse anti-CCR9 antibody. Total RNA was isolated and quantitative RT-PCR analysis was performed for mRNA expression of MMPs and transcript copies are presented relative to actual copies of $18 \mathrm{~S}$ rRNA. Active protein expression was quantified by Fluorokine and Biotrak assays in conditioned media. "Statistical differences $(\mathrm{P}<0.01)$ between untreated and CCL25-treated BrCa cells.

gradients of CCL25 (Fig. 3). The CCR9 ${ }^{\mathrm{Lo}} \mathrm{MCF}-7$ cells were largely unresponsive to CCL25. The number of MDA-MB231 cells that migrated in response to CCL25 was significantly higher than untreated cells. This chemokine receptordependent interaction was neutralized by treatment with antiCCR9 antibody. Additionally, the invasiveness of MDAMB-231 and MCF-7 cell lines was assessed after CCL25 treatment. The ability to invade Matrigel by MDA-MB-231 cells, which expressed the highest levels of CCR9, was increased following stimulation with CCL25 (Fig. 4). As expected, this invasive phenotype was abrogated following CCR9 neutralization. MCF-7 cells were not significantly invasive in response to CCL25. Taken together, MDA-MB231, but not MCF-7, cells migrated and invaded in response to CCL25 in a CCR9-dependent fashion.

CCL25-induced MMP expression. The Matrigel system used in the invasion assays consisted of laminin, collagen IV and enactin; hence, MMP-1, MMP-2, MMP-3, MMP-9, MMP-10, MMP-11 and/or MMP-13 may be required to optimally digest these components. To determine whether the associated increase in cancer cell invasiveness after CCR9-CCL25 interaction was due to higher MMP expression, collagenase (MMP-1 and -13), gelatinase (MMP-2 and -9), and strome-
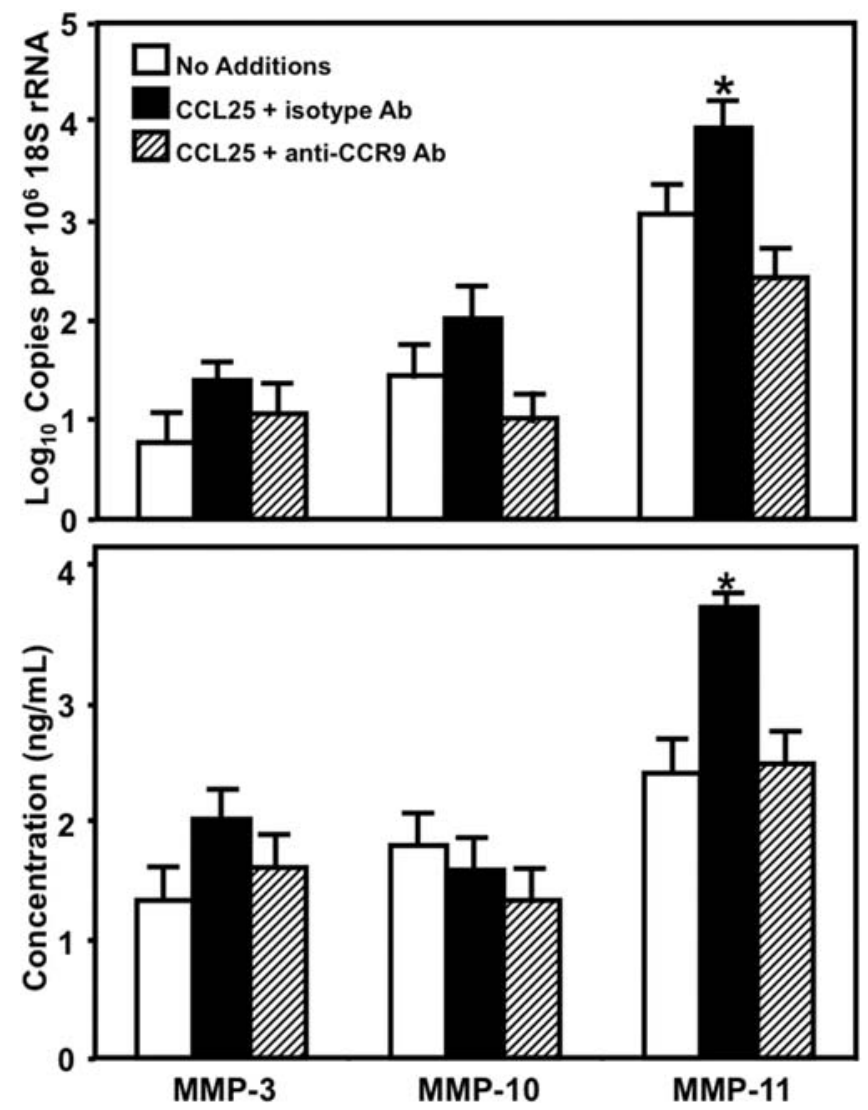

Figure 6. CCL25-induced stromelysin expression by breast cancer cells Cells were tested for their ability to express stromelysins (MMP-3, MMP-10 and MMP-11) mRNA and active protein. MDA-MB-231 cells were cultured for $24 \mathrm{~h}$ alone, with $100 \mathrm{ng} / \mathrm{ml}$ of CCL25 or CCL25 + $1.0 \mu \mathrm{g} / \mathrm{ml}$ of mouse anti-CCR9 antibody. Total RNA was isolated and quantitative RT-PCR analysis was performed for mRNA expression of MMPs and transcript copies are presented relative to actual copies of $18 \mathrm{~S}$ rRNA. Active protein expression was quantified by Fluorokine and Biotrak assays in conditioned media. *Statistical differences $(\mathrm{P}<0.01)$ between untreated and CCL25-treated BrCa cells.

lysin (MMP-3, -10 and -11) mRNA expression was assessed by quantitative RT-PCR analysis and functional protein expression was assessed using MMP activity assays. Both untreated and CCL25-treated MDA-MB-231 cells expressed collagenases (Fig. 4). When compared to untreated cells, CCL25-treated cells expressed significantly higher levels of active MMP-1 protein. Similarly, the levels of MMP-13 mRNA levels and activities were significantly increased following CCL25 treatment and CCR9 blockade abrogated these events. MDA-MB-231 cells also expressed gelatinases (Fig. 5). MDA-MB-231 cells modestly upregulated MMP-2 mRNA and active protein expression following CCL25 treatment. CCL25-treated MDA-MB-231 cells expressed significantly higher levels of MMP-9 mRNA than compared to untreated cells. CCL25 also greatly increased active MMP-9 expression. CCR9 blocked inhibited both MMP-9 mRNA and active protein expression increase caused by CCL25. Neither MMP-3 or MMP-10 mRNA or active protein levels were modulated by CCL25. However, CCL25 treatment of MDA-MB-231 cells induced a significant increase in MMP-11 mRNA and active protein expression than were CCR9-dependent (Fig. 6). Together, the data show that 
CCL25-mediates collagenase, gelatinase and stromelysin mRNA and active protein expression levels in a CCR9dependent fashion.

\section{Discussion}

Chemokines have been shown to be involved in lymphocyte chemotaxis $(6,7)$, and cause cancer cells to home to specific secondary sites to promote organ-specific metastasis $(8,9)$. It has recently been shown that CXCR4, CXCR7 and CCR7 are expressed by $\mathrm{BrCa}$ cells (10-13) and play an important role in metastatic spread of breast tumor cells to the lungs and lymph nodes $(5,14)$. CCR9-CCL25 interactions have been implicated in the pathogenesis and progression of melanoma (15) and prostate cancers (9). CCR9 has been shown to be highly expressed by double-positive thymocytes and CCL25 causes chemotaxis of these cells $(16,17)$. Indeed, CCL25 is expressed by the thymus, small intestines, and at low levels in the spleen (18), allowing CCR9-positive cells to preferentially home to the lamina propia of the small bowel and intraepithelial compartments (19). In general, metastases of $\mathrm{BrCa}$ frequently occur in the lungs, liver, adrenals and bone $(20,21)$. However, autopsies of $\mathrm{BrCa}$ show small intestinal metastases in $16 \%$ of $\mathrm{BrCa}$ cases $(22,23)$. Hence, intestinal metastasis is an under-diagnosed complication of late stage $\mathrm{BrCa}$.

We have shown for the first time that $\mathrm{BrCa}$ tissues and cell lines significantly express CCR9. BrCa tissues display the strongest CCR9 immunointensity in poorly differentiated carcinomas. CCR9 expression is functional, as indicated by CCL25-mediated BrCa cell migration and invasion, perhaps this mechanism support $\mathrm{BrCa}$ cell spread to the lymph nodes and gastrointestinal tract, which are known to selectively express CCL25 (24). MDA-MB-231 cells expressed higher levels of CCR9 and had a greater migration and invasion response to CCL25, than compared to the less invasive MCF-7 cell line. To this end, others have shown that MDA-MB-231 cells are more invasive than MCF-7 cells $(25,26)$.

In order for malignant tumors to initiate the metastatic process, neoplastic cells must penetrate the basement membrane, invade through the interstitial stroma and survive in the circulation as well as at the new metastatic site. CCR9CCL25 interactions have been shown to play a role in antiapoptosis (27). Hence, these chemokine-chemokine receptor axis supports $\mathrm{BrCa}$ cell survival in metastatic sites as well as degradation of extracellular matrix (ECM) components for primary tumor cell escapes or increase of secondary sites.

The over-expression of MMP-1 has been indicated in the poor prognosis of colorectal $(28,29)$, esophageal $(30)$ and bladder $(31,32)$ cancers. MMP-1 has also been shown to be expressed by breast tumor tissue and MDA-MB-231 cells under serum-free conditions (33). We show that MDA-MB231 cells expressed MMP-1 messenger RNA and active protein, and this expression was modulated by CCL25 in a CCR9-dependent fashion. MMP-13, which is another important collagenase required for the degradation of type I and II collagens, was elevated after CCR9 activation of $\mathrm{BrCa}$ cells. While this MMP has not been shown to have a major role in $\mathrm{BrCa}$ to date, MMP-13 plays a crucial role in the invasiveness of head and neck cancers (34).
MMP-2 and -9 are type IV collagenases, which degrade gelatin, collagen and other basement membrane components. Our results show that CCL25 increased MMP-9, but not MMP-2, mRNA and active protein expression, which was dependent on CCR9. While MMP-2 has previously been shown to be associated with the high potential of metastasis in many carcinomas, including $\mathrm{BrCa}$ (35), the expression of MMP-9 plays a significant role in metastasis, angiogenesis, and tumor progression (36). Stromelysins (MMP-3, -10 and -11) are predominantly expressed by normal epithelial cells, but are frequently over-expressed by carcinomas, where they degrade a broad range of substrates, including type IV, V, IX and $\mathrm{X}$ collagens, fibronectins, laminin, elastin, gelatin and proteoglycan core proteins (37). Like MMP-9, MMP-3 is thought to play an important role in $\mathrm{BrCa}$ invasion, metastasis and tumor angiogenesis. Increased expression of stromelysins correlates with poor prognosis and are detected more frequently in malignant rather than benign breast tumors. It has been suggested that MMP-3 is involved in brain metastasis of $\mathrm{BrCa}$ (38). In this study, CCL25 treatment of MDA-MB-231 cells induced a large increase in MMP-11 mRNA, but not MMP-3 or MMP-10, transcripts. CCR9CCL25 interactions also increased active MMP-11 expression. MMP-11 was originally identified as a $\mathrm{BrCa}$-associated gene and has been shown to be overexpressed in $>90 \%$ of invasive breast carcinomas (39). Its expression has been shown to have a negative effect on patients' survival (40) and may play a role in the metastasis of $\mathrm{BrCa}$ cells i.e., their homing to and survival in bone (41). In summary, we provide the first evidence that $\mathrm{BrCa}$ cells express functional CCR9. The effect of CCL25 on BrCa cell migration, invasion and MMP expression suggests that this chemokine may play a significant role in $\mathrm{BrCa}$ progression. Additional studies will be necessary to evaluate the precise mechanisms of CCL25and CCR9-mediated migration and invasion of $\mathrm{BrCa}$ cells.

\section{Acknowledgments}

The content of this manuscript benefited from many fruitful conversations with members of the Morehouse School of Medicine and the University of Alabama at Birmingham. This study was supported by National Institute of Health grants (CA092078, CA086359, DK58967, GM08248, MD00525 and RR03034) and by the Research Centers in Minority Institutions Program (RCMI)-funded Flow cytometry and cell sorting core at the Morehouse School of Medicine.

\section{References}

1. Gonzalez-Angulo AM, Morales-Vasquez F and Hortobagyi GN: Overview of resistance to systemic therapy in patients with breast cancer. Adv Exp Med Biol 608: 1-22, 2007.

2. Nicolson GL: Organ specificity of tumor metastasis: role of preferential adhesion, invasion and growth of malignant cells at specific secondary sites. Cancer Metastasis Rev 7: 143-188, 1988.

3. Chambers AF, Groom AC and MacDonald IC: Dissemination and growth of cancer cells in metastatic sites. Nat Rev Cancer 2: 563-572, 2002.

4. Patanaphan V, Salazar OM and Risco R: Breast cancer: metastatic patterns and their prognosis. South Med J 81: 1109-1112, 1988 . 
5. Muller A, Homey B, Soto H, et al: Involvement of chemokine receptors in breast cancer metastasis. Nature 410: 50-56, 2001

6. David R and Marelli-Berg FM: Regulation of T-cell migration by co-stimulatory molecules. Biochem Soc Trans 35: 1114-1118, 2007.

7. Trentin L, Miorin M, Facco M, et al: Multiple myeloma plasma cells show different chemokine receptor profiles at sites of disease activity. Br J Haematol 138: 594-602, 2007.

8. Gomperts BN and Strieter RM: Chemokine-directed metastasis. Contrib Microbiol 13: 170-190, 2006.

9. Singh S, Singh UP, Grizzle WE and Lillard JW: CXCL12CXCR4 interactions modulates prostate cancer cell migration, metalloproteinase expression and invasion. Lab Invest 84: 1666-1676, 2004.

10. Cabioglu N, Gong Y, Islam R, et al: Expression of growth factor and chemokine receptors: new insights in the biology of inflammatory breast cancer. Ann Oncol 18: 1021-1029, 2007.

11. Cabioglu N, Yazici MS, Arun B, et al: CCR7 and CXCR4 as novel biomarkers predicting axillary lymph node metastasis in T1 breast cancer. Clin Cancer Res 11: 5686-5693, 2005.

12. Moore MA: The role of chemoattraction in cancer metastases. Bioessays 23: 674-676, 2001

13. Miao Z, Luker KE, Summers BC, et al: CXCR7 (RDC1) promotes breast and lung tumor growth in vivo and is expressed on tumor-associated vasculature. Proc Natl Acad Sci USA 104: 15735-15740, 2007.

14. Meijer J, Ogink J and Roos E: Effect of the chemokine receptor CXCR7 on proliferation of carcinoma cells in vitro and in vivo. Br J Cancer 99: 1493-1501, 2008.

15. Hwang ST: Chemokine receptors in melanoma: CCR9 has a potential role in metastasis to the small bowel. J Invest Dermatol 122: xiv-xv, 2004

16. Wurbel MA, Malissen M, Guy-Grand D, et al: Mice lacking the CCR9 CC-chemokine receptor show a mild impairment of early $\mathrm{T}$ - and $\mathrm{B}-$-cell development and a reduction in T-cell receptor gammadelta $(+)$ gut intraepithelial lymphocytes. Blood 98 2626-2632, 2001.

17. Wurbel MA, Philippe JM, Nguyen C, et al: The chemokine TECK is expressed by thymic and intestinal epithelial cells and attracts double- and single-positive thymocytes expressing the TECK receptor CCR9. Eur J Immunol 30: 262-271, 2000.

18. Papadakis KA, Prehn J, Nelson V, et al: The role of thymusexpressed chemokine and its receptor CCR9 on lymphocytes in the regional specialization of the mucosal immune system. J Immunol 165: 5069-5076, 2000

19. Elgueta R, Sepulveda FE, Vilches F, et al: Imprinting of CCR9 on CD4 T cells requires IL-4 signaling on mesenteric lymph node dendritic cells. J Immunol 180: 6501-6507, 2008.

20. Asch MJ, Wiedel PD and Habif DV: Gastrointestinal metastases from carcinoma of the breast. Autopsy study and 18 cases requiring operative intervention. Arch Surg 96: 840-843, 1968.

21. Birla R, Mahawar KK, Orizu M, Siddiqui MS and Batra A Caecal metastasis from breast cancer presenting as intestinal obstruction. World J Surg Oncol 6: 47, 2008.

22. Cifuentes N and Pickren JW: Metastases from carcinoma of mammary gland: an autopsy study. J Surg Oncol 11: 193-205, 1979.

23. Hata K, Kitayama J, Shinozaki M, et al: Intestinal perforation due to metastasis of breast carcinoma, with special reference to chemotherapy: a case report. Jpn J Clin Oncol 31: 162-164, 2001.

24. Carramolino L, Zaballos A, Kremer L, et al: Expression of CCR9 beta-chemokine receptor is modulated in thymocyte differentiation and is selectively maintained in $\mathrm{CD} 8^{+} \mathrm{T}$ cells from secondary lymphoid organs. Blood 97: 850-857, 2001.
25. Fox BP and Kandpal RP: Invasiveness of breast carcinoma cells and transcript profile: Eph receptors and ephrin ligands as molecular markers of potential diagnostic and prognostic application. Biochem Biophys Res Commun 318: 882-892, 2004.

26. Fritah A, Saucier C, De Wever O, et al: Role of WISP-2/CCN5 in the maintenance of a differentiated and noninvasive phenotype in human breast cancer cells. Mol Cell Biol 28: 1114-1123, 2008.

27. Youn BS, Yu KY, Oh J, Lee J, Lee TH and Broxmeyer HE: Role of the CC chemokine receptor 9/TECK interaction in apoptosis. Apoptosis 7: 271-276, 2002.

28. Lievre A, Milet J, Carayol J, et al: Genetic polymorphisms of MMP1, MMP3 and MMP7 gene promoter and risk of colorectal adenoma. BMC Cancer 6: 270, 2006

29. Woo M, Park K, Nam J and Kim JC: Clinical implications of matrix metalloproteinase- $1,-3,-7,-9,-12$, and plasminogen activator inhibitor-1 gene polymorphisms in colorectal cancer. J Gastroenterol Hepatol 22: 1064-1070, 2007.

30. Gu ZD, Li JY, Li M, et al: Matrix metalloproteinases expression correlates with survival in patients with esophageal squamous cell carcinoma. Am J Gastroenterol 100: 1835-1843, 2005.

31. Kader AK, Liu J, Shao L, et al: Matrix metalloproteinase polymorphisms are associated with bladder cancer invasiveness. Clin Cancer Res 13: 2614-2620, 2007.

32. Nutt JE, Mellon JK, Qureshi K and Lunec J: Matrix metalloproteinase- 1 is induced by epidermal growth factor in human bladder tumour cell lines and is detectable in urine of patients with bladder tumours. Br J Cancer 78: 215-220, 1998.

33. Nutt JE and Lunec J: Induction of metalloproteinase (MMP1) expression by epidermal growth factor (EGF) receptor stimulation and serum deprivation in human breast tumour cells. Eur J Cancer 32A: 2127-2135, 1996

34. Culhaci N, Metin K, Copcu E and Dikicioglu E: Elevated expression of MMP-13 and TIMP-1 in head and neck squamous cell carcinomas may reflect increased tumor invasiveness. BMC Cancer 4: 42, 2004

35. Li HC, Cao DC, Liu Y, et al: Prognostic value of matrix metalloproteinases (MMP-2 and MMP-9) in patients with lymph node-negative breast carcinoma. Breast Cancer Res Treat 88: 75-85, 2004.

36. Mira E, Lacalle RA, Buesa JM, et al: Secreted MMP9 promotes angiogenesis more efficiently than constitutive active MMP9 bound to the tumor cell surface. J Cell Sci 117: 1847-1857, 2004.

37. Shapiro SD: Matrix metalloproteinase degradation of extracellular matrix: biological consequences. Currt Opin Cell Biol 10: 602-608, 1998 .

38. Mendes O, Kim HT and Stoica G: Expression of MMP2, MMP9 and MMP3 in breast cancer brain metastasis in a rat model. Clin Exp Metastasis 22: 237-246, 2005.

39. Basset P, Bellocq JP, Wolf C, et al: A novel metalloproteinase gene specifically expressed in stromal cells of breast carcinomas. Nature 348: 699-704, 1990.

40. Nakopoulou L, Panayotopoulou EG, Giannopoulou I, et al: Stromelysin-3 protein expression in invasive breast cancer: relation to proliferation, cell survival and patients' outcome. Mod Pathol 15: 1154-1161, 2002.

41. Delany AM and Canalis E: The metastasis-associated metalloproteinase stromelysin-3 is induced by transforming growth factor-beta in osteoblasts and fibroblasts. Endocrinology 142: $1561-1566,2001$ 